\section{Förderpreis für Arbeitsgemeinschaft Asthmaschulung}

m Rahmen ihrer Jahrestagung hat die

Gesellschaft für Pädiatrische Allergologie und Umweltmedizin (GPA) e.V. am 2. September 2006 in Hamburg den „Förderpreis Pädiatrische Allergologie 2006 " verliehen. Den mit $5.000 €$ dotierten Preis konnte die Arbeitsgemeinschaft Asthmaschulung im Kindes- und Jugendalter (AGAS) e. V. aus Osnabrück entgegennehmen. Mit der von Phadia, Freiburg, gestifteten Auszeichnung werden einmal jährlich Projekte gewürdigt, die in besonderer Weise zur Verbesserung der Situation von allergiekranken Kindern beigetragen haben. Der erste Vorsitzende der GPA, Prof. Dr. Carl Peter Bauer, Gaißach, überreichte den Preis an den Vorsitzenden der AGAS, Dr. Rüdiger Szczepanski aus Osnabrück, der damit auch für sein persönliches Engagement gewürdigt wurde.

Asthma bronchiale stellt die häufigste chronische Erkrankung im Kindesund Jugendalter dar. Die Asthmaschulung wird dabei zunehmend als wesent- licher Baustein der Behandlung anerkannt. Die strukturierte Asthmaschulung nach den Qualitätskriterien der AGAS ist als einziges Programm im Rahmen des Disease-Management-Programms Asthma bronchiale zugelassen. Innerhalb der AGAS sind viele Berufsgruppen wie Ärzte, Psychologen, Päda-

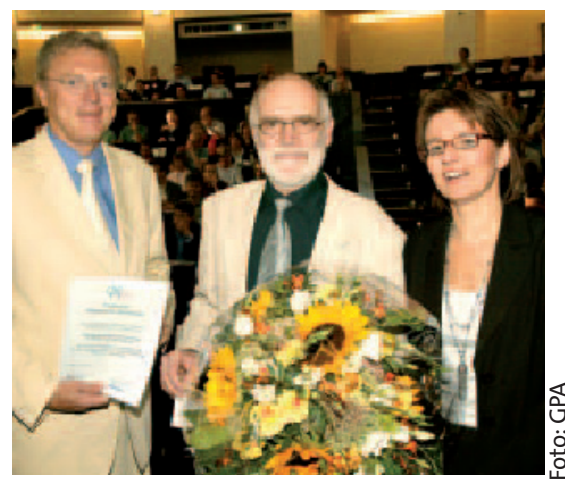

Von links nach rechts: Prof. Dr. Carl Peter Bauer, Erster Vorsitzender der GPA, Dr. Rüdiger Szczepanski, Vorsitzender der AGAS, und Kerstin Kaniecki-Loop, Phadia gogen, Kinderkrankenpflegepersonal und Physiotherapeuten für die Asthmaschulung tätig. Diese Zusammensetzung spiegelt wider, wie wichtig Teamarbeit bei der praktischen Durchführung der Schulung ist. Die Asthmaschulung der AGAS versteht sich als pädagogisch-psychologische Maßnahme, um alltagstaugliches Wissen an Kinder, Jugendliche und deren Familien zu vermitteln. Zentrale Elemente sind dabei Verhaltenstraining, die Verbesserung der eigenen Körperwahrnehmung, der Umgang mit körperlich empfundenen Symptomen, eine Verbesserung des gefühlsmäßigen Umgangs und auch eine Verringerung negativer Krankheitsauswirkungen im familiären sowie sozialen Bereich.

Seit ihrer Gründung im Jahre 1994 hat die AGAS entscheidenden Anteil an der Verbesserung der Patientenversorgung. Inzwischen gibt es bundesweit über 2.500 qualifizierte Asthmatrainer. Ziele der Arbeitsgemeinschaft sind neben der Sicherung des erreichten Standards bei der Schulung die Ausbildung weiterer kompetenter Asthmatrainer und damit ein flächendeckendes Schulungsangebot.

$M M$

\section{Studienaufruf „Stellenwert der Atemphysiotherapie bei COPD“}

Die Atemphysiotherapie ist eine unterstützende Säule in der Behandlung von COPD-Patienten. Die Patienten sollen von befundorientierten Behandlungstechniken und erlernten Selbsthilfemaßnahmen profitieren. Leider gibt es bisher keine methodisch einwandfreien Studien, die die Effekte der Atemphysiotherapie bei COPDPatienten nachweisen. So sind viele Techniken derzeit nur mit Evidenzgrad C belegt.

Vor diesem Hintergrund plant die Arbeitsgemeinschaft Atemtherapie im Zentralverband der Physiotherapeuten/Krankengymnasten in Zusammenarbeit mit der Deutschen Atemwegsliga e.V. die Wirksamkeit der ambulanten Atemphysiotherapie zu evaluieren. In der Studie „Stellenwert der Atemphysiotherapie in der Behandlung von COPD-Patienten mit Husten " wird für die Patienten mit Husten eine Verbesserung in verschiedenen Bereichen erwartet. Der Nachweis soll mit standardisierten Verfahren und Tests geführt werden. Durchgeführt wird eine multizentrische randomi- sierte plazebokontrollierte Studie mit drei Therapiearmen. Insgesamt 210 Patienten werden zugeteilt auf

- Gruppe „blau“: Standardtherapie und Add-on-Physiotherapie

_ Gruppe „violett“: Standardtherapie und Add-on-Plazebo-Physiotherapie

- Gruppe „grün“: Standardtherapie („usual care") und Behandlung in 25 bis 30 deutschen Physiotherapiepraxen mit Schwerpunkt Atemphysiotherapie.

\section{Einschlusskriterien:}

— Diagnose COPD (Anamnese + Untersuchung: $\mathrm{FEV}_{1} / \mathrm{VK}<70 \%$, $\mathrm{FEV}_{1}$ postbronchodilatatorisch $<50 \%$ Soll und $\Delta \mathrm{FEV}_{1}<15 \%$ nach Inhalation von $200 \mu \mathrm{g}$

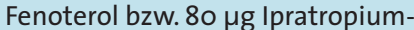
bromid)

— Subjektive Beeinträchtigung durch Husten

\section{Ausschlusskriterien :}

- Atemphysiotherapie (incl. Flutter und Cornet) zur Behandlung der COPD innerhalb der letzten sechs Monate
Exazerbation innerhalb der letzten vier Wochen; Einschluss nach Ende der Exazerbation bei einer Wartezeit von vier Wochen möglich

— Änderung der COPD Dauertherapie in den letzten vier Wochen

—Zusätzliche bedeutsame Lungenkrankheit wie z. B. bekannte Bronchiektasie oder Lungenkarzinom

_ Immobilisation, Herzinsuffizienz (NYHA III, IV), Demenz

Die Deutsche Atemwegsliga bittet um Unterstützung bei dieser wichtigen Studie. Studienkoordinator Dr. Oliver Göhl beantwortet gerne weitere Fragen oder nimmt Ihre Anmeldung entgegen. Pro Patient, den Sie rekrutieren, erhalten Sie eine Aufwandsentschädigung in Höhe von $50,-€$.

\section{Dr. Oliver Göhl}

Koordinator der Studie „Stellenwert der Atemphysiotherapie in der Behandlung von COPD-Patienten mit Husten" Tel.: (og 11) 9615411

E-Mail: olivergoehl@nefkom.net 